\title{
An Improved Localization Algorithm for Error Minimization in Wireless Sensor Networks
}

\author{
Santar Pal Singh ${ }^{1 *}$, S. C. Sharma ${ }^{1}$, \\ ${ }^{1}$ Electronics and Computer Discipline, DPT, IIT Roorkee, India, 247667 \\ spsingh78@gmail.com
}

\begin{abstract}
Wireless sensor networks (WSNs) are comprised of huge number of resource constrained petite devices recognized as sensor nodes. Data collection and forwarding is an important function of WSN. In the majority of applications, the location of the data is of much significance. Such kind of info can be acquired by using localization schemes. So, node localization is extremely essential to determine the location of node by using localization algorithms. Hence, localization becomes a hot research issue in WSNs. Many localization algorithms have been projected to precisely locate nodes in WSNs. These algorithms are broadly classified into range based and range free categories. Range based methods use exact computation measure (distance or time) among nodes in the network. So range based methods needs some additional hardware for such computation while range free methods don't require any specific hardware. Range free methods use connectivity information between nodes in network. So range free methods are considered cost effective alternatives to range based methods. In this paper, we propose a novel range free localization algorithm for WSN on the basis of swarm intelligence. We perform the comparative analysis of proposed algorithm with DV-Hop algorithms on the basis of localization error and accuracy. Simulation results shows that proposed algorithm outperform the DV-Hop algorithm.
\end{abstract}

Keyword- wireless sensor network, localization algorithms, DV Hop, error, accuracy

\section{INTRODUCTION}

Latest advancement in MEMS and wireless communication technologies allows the micro autonomous system comprised of small tiny devices known as sensors. These sensors can detect, compute and communicate via suitable sensor technology that gives birth to wireless sensor network [1]-[3]. Deployment ease and low cost sensors make wireless sensor network suitable for many applications like: health care, transportation, smart building, and environmental monitoring etc. For such applications the physical location of sensor are needed to detect the events in monitored area [3]-[4]. So the location information is of much use in various applications and needed to build up context aware applications [4].

The majority of WSN applications used a model where some of the nodes are ware about their location (either manually placed or enabled with GPS) are known as anchors or beacon nodes. Remaining nodes are normal nodes, randomly deployed due to the hostility of the region to be kept an eye on. The nodes compute their locations with the help of information they received from the anchors [5]-[9]. But this model have some drawbacks: expensive method (due to cost of GPS), inaccurate in indoor environment and large scale deployment of WSN [10]-[11]. Many algorithms have been developed for node localization [12]-[16]. Based on the range's assessment, the localization algorithms are broadly categorizes as: range based and range free. The ranging methods used accurate value of distance or angle as range measurements. Several ranging methods are possible for range measurements like: signal strength based (RSSI), Time based (ToA/TDoA), and angle based (AoA) etc [14]-[15]. Because of certain hardware limitations and offset range errors, researchers designed range free schemes that are based on proximity or connectivity information measures [16]-[21]. The popular methods of these schemes are Centroid, APIT, Amorphous, and DV-Hop etc.

In this paper, we proposed a modified algorithm for localization on the basis of distance vector hop algorithm. The proposed method reduces the localization error and improves accuracy without raising the cost of the sensor node. Here the presented work contributes three most important things to localization problem. First, we propose a new modified algorithm that is easy to use and cost effective. Second, the proposed algorithm reduces the localization error as compared to DV-Hop. Third, we explore the impact of anchors and communication range on performance of new modified algorithm. The remaining part of paper is planned as; section 2 focused on a literature review. Section 3 focused on the DV Hop localization algorithms. The proposed algorithm is illustrated in section 4. Section 5 focused on the result and discussion of performance of algorithms. Finally, section 6 concludes the work. 


\section{RELATED WORK}

Here, we assess the literature appropriate to the work. In recent past, many works propose the schemes for localization in WSNs. Since every method was designed to solve a dissimilar objective, they differ extensively in parameters includes size, cost, accuracy, and reliability. The examples are: RADAR, Active Bats, SpotON, and Calamari etc [14]-[15]. The range free algorithms are slightly influenced by some environmental factors but additional ranging hardware not required. Such types of characteristics make them appropriate for WSN applications. In range free category, the Niculescu et al. proposes a distance vector hop based schemes know as DV-Hop scheme [16]. In this method, the sensor nodes initially count the value of minimal hop from anchor nodes and afterward estimate the distance amid anchors and unknown nodes. At last, the sensor nodes find out their locations with help of angulations' or maximum likelihood estimation (MLE). He et al devised a new area based method known an APIT [17]. With help of three beacons, APIT (Approximate Point in Triangle Test) used a method based on area to compute the node positions. Centroid is an easy algorithm for localization. In this, nodes obtain the landmarks signals in its range and make its coordinate as centroid. Amorphous is alike to distance vector hop based method, however it likely to identify the node's density a prior with help of offline estimation of hop distance [20]. It is supposed to make a relatively accurate method via local information. The Centroid, APIT, Amorphous, and DV-Hop are distributed range free localization algorithms. So, they are outlined by low traffic and computational simplicity. In the recent past, based on various improved schemes were proposed on the basis of DV-Hop method.

\section{DV-HOP LOCALIZATION ALGORITHM}

All paragraphs must be indented. The DV-Hop method is designed by Niculesue [16]. The main idea behind this is that nodes exchange information from its neighbors. This algorithm requires that some nodes are aware about their location (either manually placed or enabled with GPS) and communication information between nodes. The nodes those are aware about their locations are recognized as beacons or anchors. The sensor nodes don't require measurement equipments so the DV-Hop is one of most applicable methods in node localization for large scale deployment of WSNs.

DV-Hop algorithm is comprised of following three steps:

Step1: Determine the minimal hop value between unknown and each anchor node.

In this step, every anchor broadcast a message all over the network comprised the location of anchor with value of hop count i.e. initially one. Every node that obtains the message records the each node's minimal hop count although ignoring the bigger one from same beacon and then hop count value is incremented by one and pass on to nearby nodes.

Step2: Determine the actual distance among unknown node and anchor node.

In this step, every anchor estimate the average hop distances by the following equation:

$$
\text { HopSize }_{i}=\frac{\sum_{i \neq j} \sqrt{\left(x_{i}-x_{j}\right)^{2}+\left(y_{i}-y_{j}\right)^{2}}}{\sum_{i \neq j} h_{i j}}
$$

Where $\left(x_{i}, y_{i}\right)$ is the location of anchor $\mathrm{i}$ and $\left(x_{j}, y_{j}\right)$ is the location of anchor $\mathrm{j} . h_{i j}$ is the hop value amid anchor $\mathrm{i}$ and anchor $\mathrm{j}$.

Anchor node disseminates the average hop value. Unknown node evidence the first collected average hops distance and pass on it to neighbours. Then unknown node determines the distance to every beacon in accordance with hop counts.

Step3: Determine the position of unknown nodes.

The unknown node use trilateration or MLE scheme to determine the coordinate of these nodes.

The distance from all beacon nodes to unknown node $\mathrm{P}\left(x_{u}, y_{u}\right)$ are given by the formula: 


$$
\begin{gathered}
\left(x_{1}-x_{u}\right)^{2}+\left(y_{1}-y_{u}\right)^{2}=d_{1}^{2} \\
\cdot \\
\cdot \\
\cdot \\
\left(x_{n}-x_{u}\right)^{2}+\left(y_{n}-y_{u}\right)^{2}=d_{n}{ }^{2}
\end{gathered}
$$

Meantime formula (2) can be expressed as:

$$
\begin{gathered}
x_{1}^{2}-x_{n}^{2}+2\left(x_{1}-x_{n}\right) x_{u}+y_{1}^{2}-y_{n}^{2}-2\left(y_{1}-y_{n}\right) y_{u}=d_{1}^{2}-d_{n}^{2} \\
x_{2}^{2}-x_{n}^{2}+2\left(x_{2}-x_{n}\right) x_{u}+y_{2}^{2}-y_{n}^{2}-2\left(y_{2}-y_{n}\right) y_{u}=d_{2}{ }^{2}-d_{n}^{2} \\
\ldots \ldots \ldots \ldots \ldots . . \\
x_{n-1}^{2}-x_{n}^{2}+2\left(x_{n-1}-x_{n}\right) x_{u}+y_{n-1}^{2}-y_{n}^{2}-2\left(y_{n-1}-y_{n}\right) y_{u}=d_{n-1}{ }^{2}-d_{n}{ }^{2}
\end{gathered}
$$

Formula (3) steps the right equation for

Where

$$
A X=B
$$

$$
\begin{aligned}
& A=\left[\begin{array}{cc}
2\left(x_{1}-x_{n}\right) & 2\left(y_{1}-y_{n}\right) \\
\cdot & \cdot \\
2\left(x_{n-1}-x_{n}\right) & 2\left(y_{n-1}-y_{n}\right)
\end{array}\right] \\
& B=\left[\begin{array}{c}
x_{1}^{2}-x_{n}^{2}+y_{1}^{2}-y_{n}^{2}+d_{1}^{2}-d_{n}^{2} \\
\ldots \\
x_{n-1}{ }^{2}-x_{n}^{2}+y_{n-1}^{2}-y_{n}^{2}+d_{n-1}^{2}-d_{n}^{2}
\end{array}\right] \\
& X=\left[\begin{array}{l}
x_{u} \\
y_{u}
\end{array}\right]
\end{aligned}
$$

The coordinate of the unknown node P are obtained with help of the following formula:

$$
P=\left(A^{T} A\right)^{-1} A^{T} B
$$

\section{IV.PROPOSED METHOD}

Here in this part of work, we propose a new algorithm by making some modification in original DV-Hop algorithm with help of PSO.

\section{A. PSO}

Particle swarm optimization (PSO) is a branch of heuristic techniques that optimizes a complex problem via repetitively attempting to get better candidate solution with respect to a specified measure of quality [22]. The flow chart representation of this method can be shown by Fig.1. 


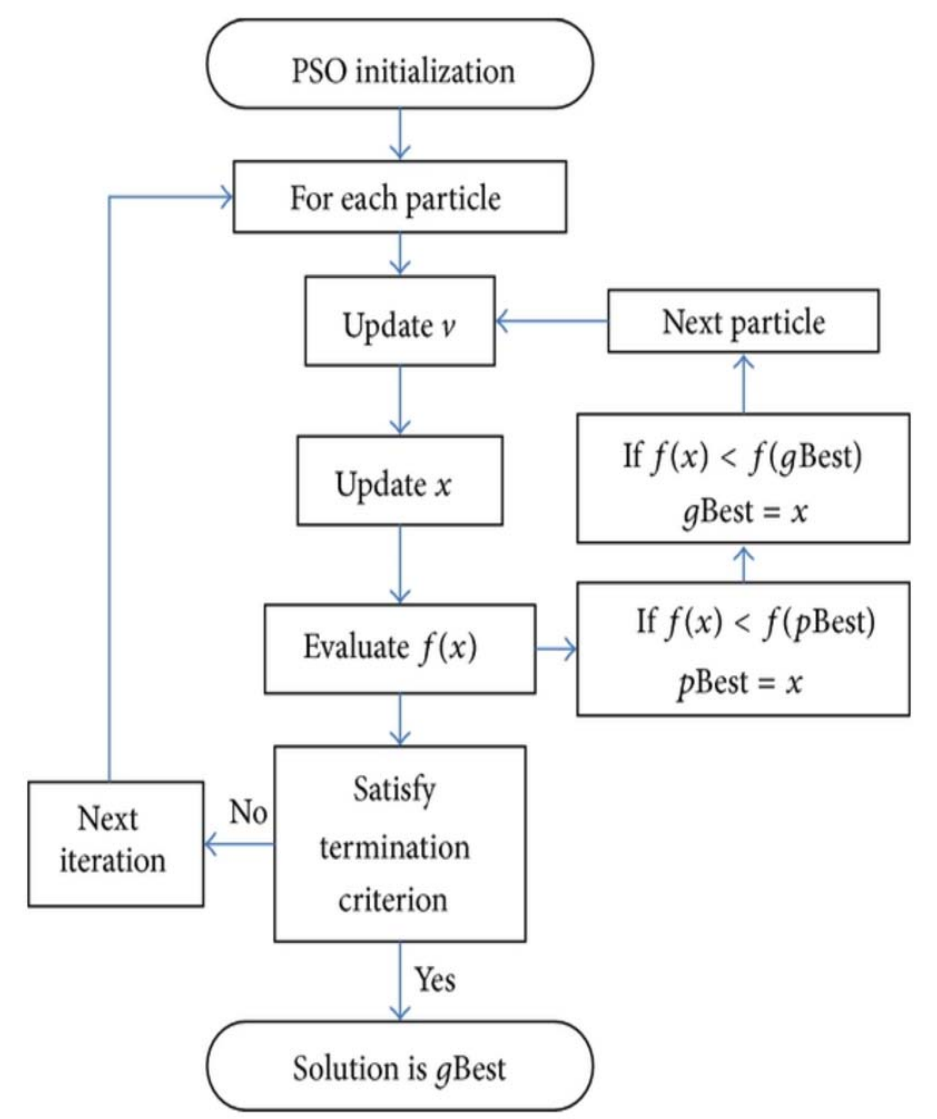

Fig. 1. Flow sheet of PSO algorithm ([31])

Updating behavior of particles can be defined as:

$$
\begin{aligned}
& v_{i}(t+1)=\omega v_{i}(t)+c_{1} r_{1}\left(\text { pbest }_{i}-x_{i}\right)+c_{2} r_{2}\left(\text { gbest }-x_{i}\right) \\
& x_{i}(t+1)=x_{i}(t)+v_{i}(t+1)
\end{aligned}
$$

Where, $v_{i}$ is the velocity of particle; $x_{i}$ is position of particle; $c_{1}$ and $c_{2}$ are the acceleration coefficients and also known as learning factors; $r_{1}$ and $r_{2}$ are the positive integers between $0 \& 1$ chosen randomly and $\omega$ is the inertia weight; pbest is individual extreme (optimal solution position of individual); gbest is global minimum (group optimal solution position of group

\section{B. Proposed Algorithm}

Title must be in $24 \mathrm{pt}$ Regular font. There are various improvements on DV-Hop algorithm is reported in literature [23-30]. Here, our modified DV-Hop algorithm at is comprised of four steps. The initial two steps of the proposed algorithm are same as of traditional DV-Hop algorithm. In step3, unknown node positions are determined with help of 2D hyperbolic algorithm. The 2D hyperbolic location algorithm improves the estimated location precision. In step 4, we use PSO to improve the estimated positions. So the step3 of the new modified algorithm is same like the improved DV-Hop algorithm. However, in the step 4 of proposed algorithm, we use PSO for correction the position estimation.

Step1: It is same as of DV-Hop method; in this step the smallest value of hops between every unknown and anchor nodes are calculated.

Step2: It is same as of DV-Hop method; in this step actual distance among unknown and anchor node is determined.

Step3: It is same as of Improved DV-Hop method; in this step, a general model for 2-D source position estimation is developed with help of anchors. Let $\left(x_{u}, y_{u}\right)$ be the location of unknown node and $\left(x_{i}, y_{i}\right)$ is the location of the $i^{\text {th }}$ anchor node and distance among them is denoted by $d_{i \text {. }}$

$d_{i}$ can be given as: 


$$
d_{i}=\sqrt{\left(x_{i}-x_{u}\right)^{2}+\left(y_{i}-y_{u}\right)^{2}}
$$

In this improved localization system, instead of conventional triangulation method, the 2-D hyperbolic location algorithm is utilized.

By the definition of above equation of $d_{i}$, we have the following expression:

$$
\begin{gathered}
x_{i}^{2}+y_{i}^{2}-2 x_{i} x_{u}-2 y_{i} y_{u}+x_{u}^{2}+y_{u}^{2}=d_{i}^{2} \\
d_{i}^{2}-E_{i}=-2 x_{i} x_{u}-2 y_{i} y_{u}+K
\end{gathered}
$$

Where $E_{i}=x_{i}^{2}+y_{i}^{2}$

$$
\begin{aligned}
K & =x_{u}{ }^{2}+y_{u}{ }^{2} \\
Z_{c} & =\left[x_{u}, y_{u}, K\right]^{T}
\end{aligned}
$$

Let

$$
G_{c}=\left[\begin{array}{ccc}
-2 x_{1} & -2 y_{1} & 1 \\
-2 x_{2} & -2 y_{2} & 1 \\
\cdot & \cdot & \cdot \\
-2 x_{i} & -2 y_{i} & 1
\end{array}\right]
$$

And

$$
H_{c}=\left[\begin{array}{c}
d_{1}^{2}-E_{1} \\
d_{2}^{2}-E_{2} \\
\cdot \\
\cdot \\
d_{i}^{2}-E_{i}
\end{array}\right]
$$

We can have

$$
G_{c} Z_{c}=H_{c}
$$

With the help of least square method, we obtain

$$
Z_{c}=\left(G_{c}^{T} G_{c}\right)^{-1} G_{c}^{T} H_{c}
$$

After that the coordinate of unknown node $\left(x_{u}, y_{u}\right)$ is given as:

$$
\begin{aligned}
& x_{u}=z_{c}(1) \\
& y_{u}=z_{c}(2)
\end{aligned}
$$

Step4: In this step, we will apply PSO to find the correct location of unknown nodes.

Let $X_{u}$ is the geographical locality of the unknown node, $B_{i}$ is the position of $i^{\text {th }}$ beacon node. The distance $d_{i}$ can be obtained as described in second step of improved DV-HOP method.

Thus the positioning error may be described as: 


$$
\begin{gathered}
f\left(X_{u}\right)=\left(d_{i}-\left|X_{u}-B_{i}\right|\right)^{2} \\
\text { fitness }\left(X_{u}\right)=\sum_{j=1}^{n}\left(\frac{1}{\text { Hop value }_{j}}\right)^{2} f\left(X_{u}\right)
\end{gathered}
$$

Particle's updations are done with the help of (6) and (7), and (15). Equation (15) is the fitness function to estimate the fitness of particles. The total no of iterations is set accordingly. After these iterations, the optimal solution is considered as the final estimated locality of the unknown node. The flow sheet representation of proposed algorithm is revealed in Fig. 2.

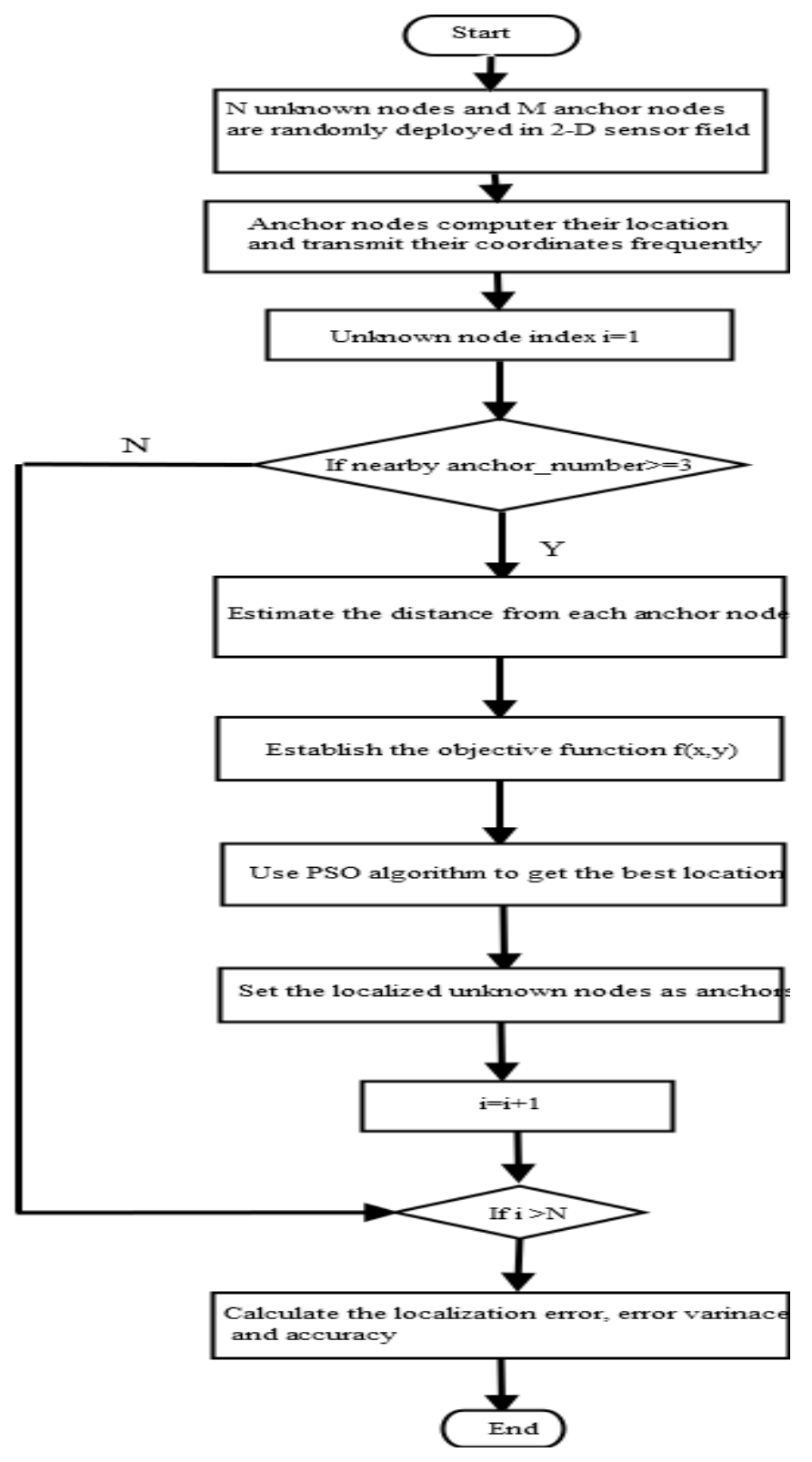

Fig. 2. Flow chart of proposed algorithm

\section{RESULTS AND DISCUSSIONS}

For the performance analysis of our modified algorithm, we use Matlab2015a as a simulator for implementation of the scenario of networks and determination of final results. Parameter setting for simulation be as: initially, few beacon nodes are fixed in $100 \times 100 \mathrm{~m}^{2}$ area then rest anchors and unknown nodes are 
distributed randomly in that area. We analyze the impact of total no of nodes, impact or anchor nodes and impact of communication range on the localization results.

The parameters in PSO algorithms are $\mathrm{c}_{1}=\mathrm{c}_{2}=2.05$, no of particles $=20, \mathrm{~V}_{\max }=10$ and we use 20 iterations. The stability and accuracy of localization are analyzed by localization error and error variance and these performance parameters are calculated by the following formula:

$$
\text { Error }_{i}=\sqrt{\left(x_{i}^{\text {eval }}-x_{i}^{\text {real }}\right)^{2}+\left(y_{i}^{\text {eval }}-y_{i}^{\text {real }}\right)^{2}}
$$

Average localization error is considered as localization error and computed as:

$$
\text { Localization Error }(L E)=\frac{\sum_{i=1}^{n} \text { Error }_{i}}{n \times R}
$$

The variance of localization error is computed as:

$$
\text { Localization Error Variance }=\frac{\sum_{i=1}^{n}\left(L E_{i}-\overline{L E}\right)^{2}}{n-1}
$$

The localization accuracy is computed as:

$$
\text { Localization Accuracy }=\frac{\sum_{i=1}^{n} \sqrt{\left(x_{i}^{\text {eval }}-x_{i}^{\text {real }}\right)^{2}+\left(y_{i}^{\text {eval }}-y_{i}^{\text {real }}\right)^{2}}}{n \times R^{2}}
$$

Wherever $n$ represent the value of unknown nodes. $x_{i}^{\text {real }}, x_{i}^{\text {eval }}$ be the real and evaluated positions of unknown node $i$ respectively. The sensor node's radio range is represented by $\mathrm{R}$.

We deploy 100 nodes out of these, few are beacon nodes and others are unknown ones, randomly in 2D area of 100 X100 m². The node's distribution is shown in Fig. 3 .

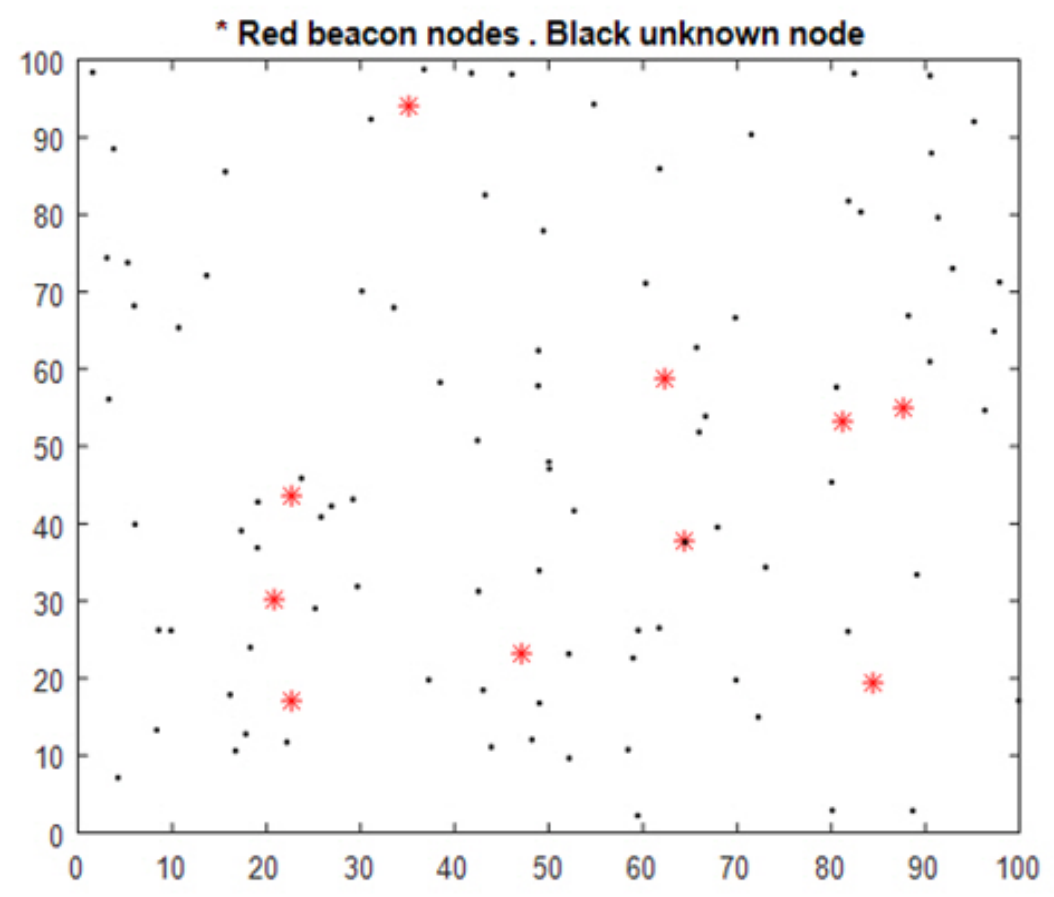

Fig. 3. Distribution of nodes 
1) Impact of number of sensor nodes on localization results

The impact of total nodes on localization parameters like error, error variance and accuracy is given away in Fig. 4, Fig.5, and Fig. 6 respectively.

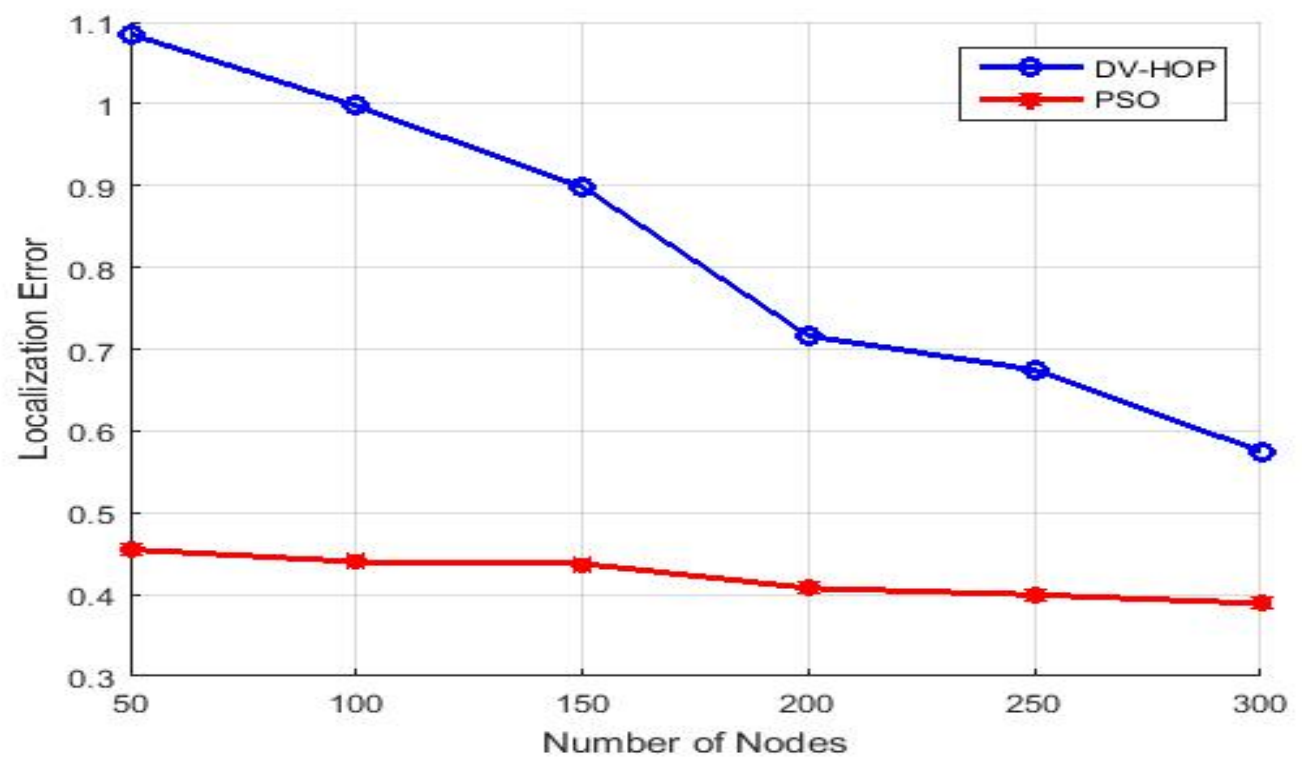

Fig. 4. Localization error with changing no. of nodes

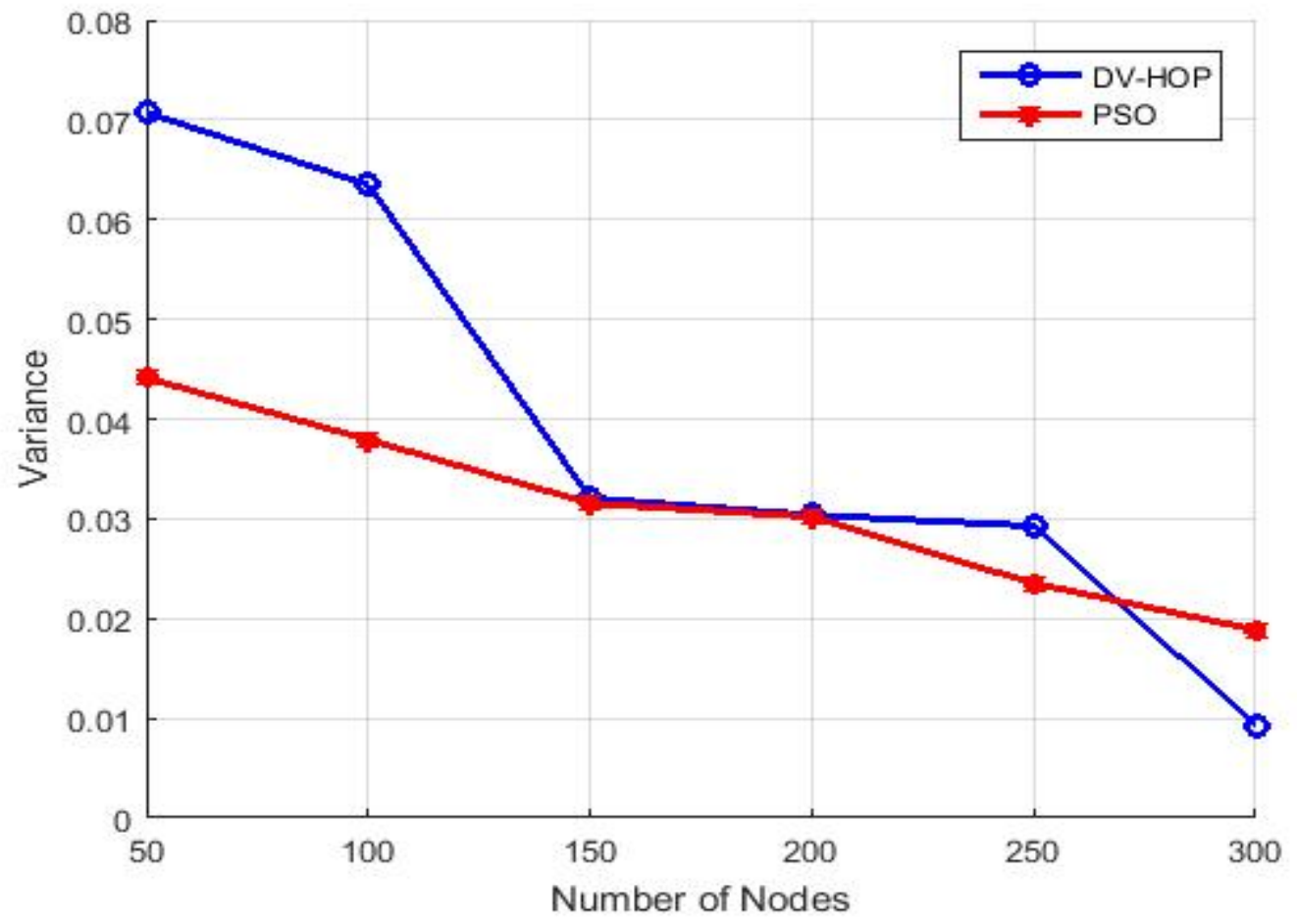

Fig. 5. Localization error variance with changing no. of nodes 


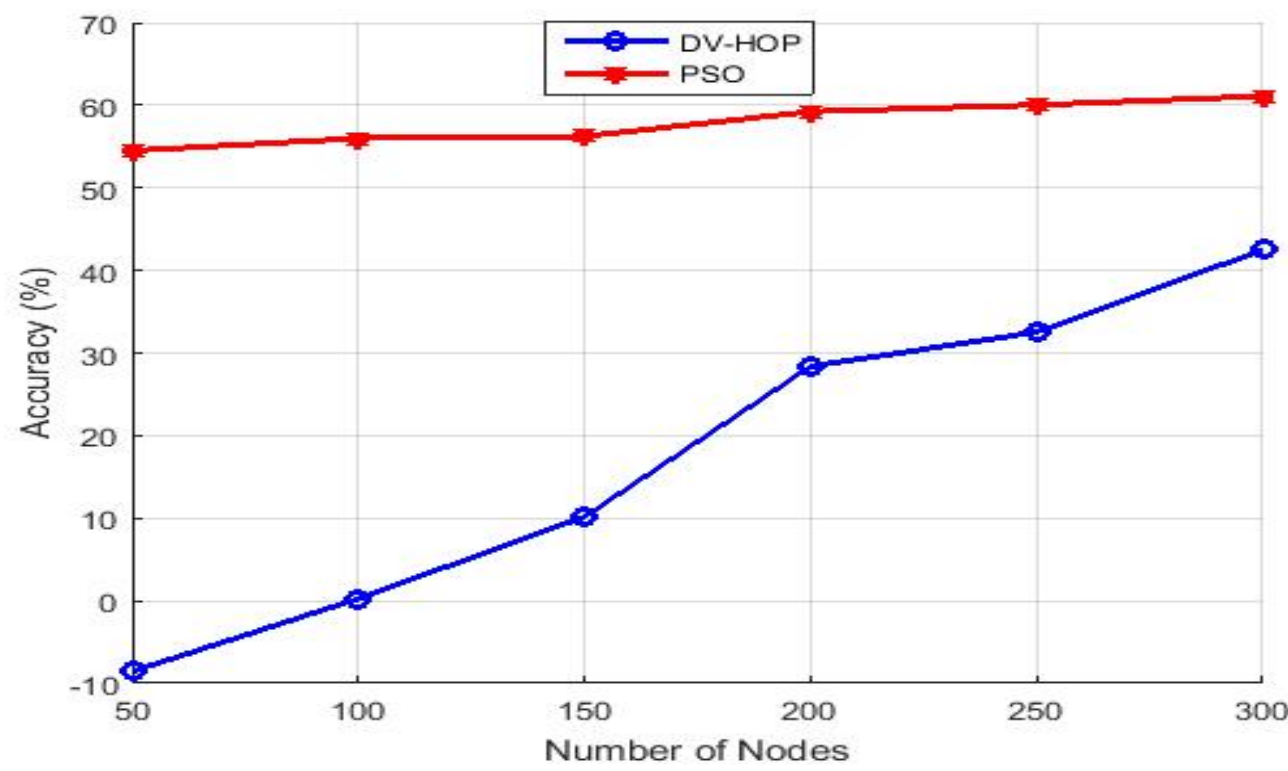

Fig. 6. Localization accuracy with changing no. of nodes

2) Impact of number of anchor nodes on localization results

The effect of beacons on the localization parameters like error, error variance and accuracy is given away in Fig. 7, Fig. 8, and Fig. 9 respectively.

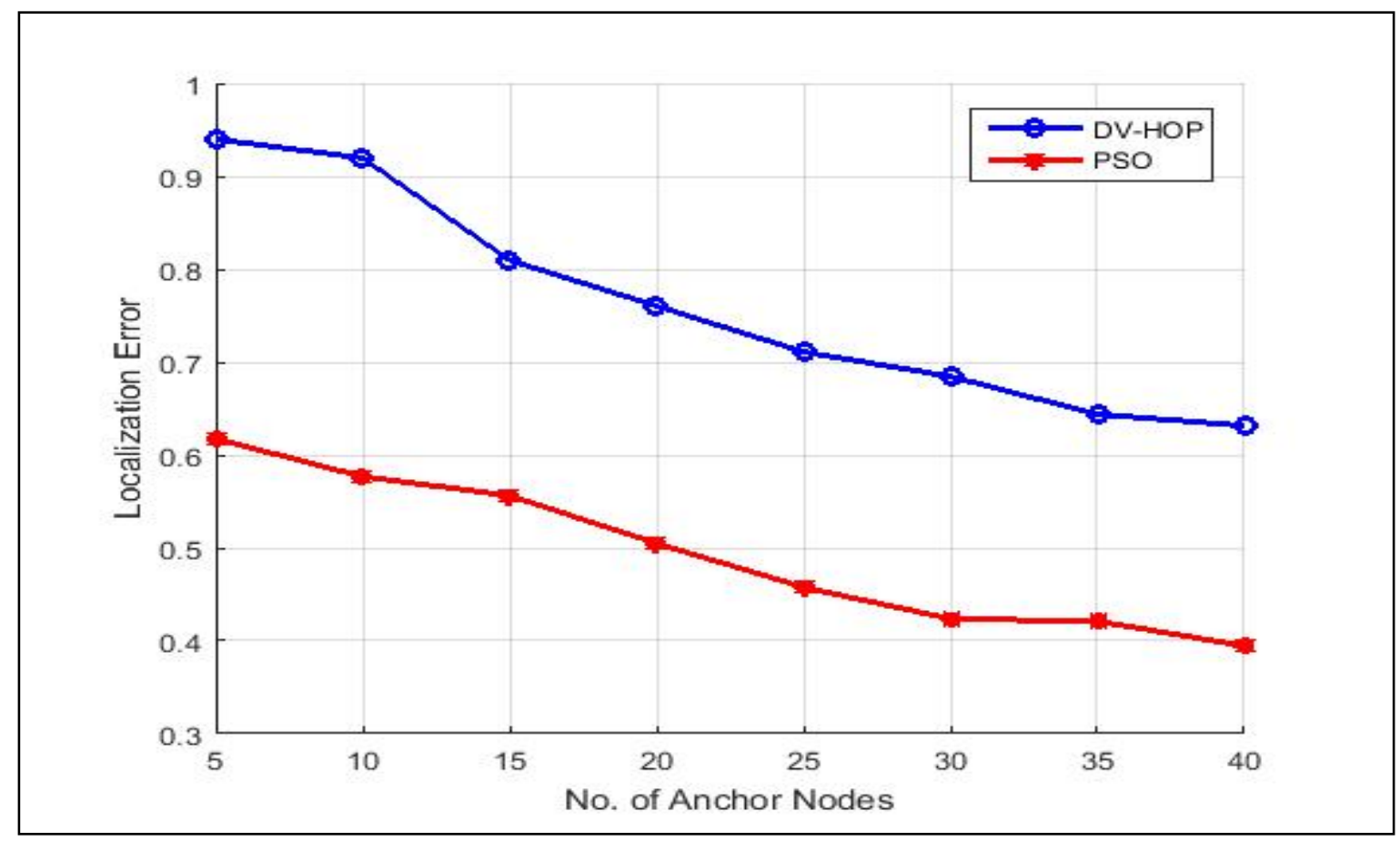

Fig. 7. Localization error with changing no. of anchor nodes 


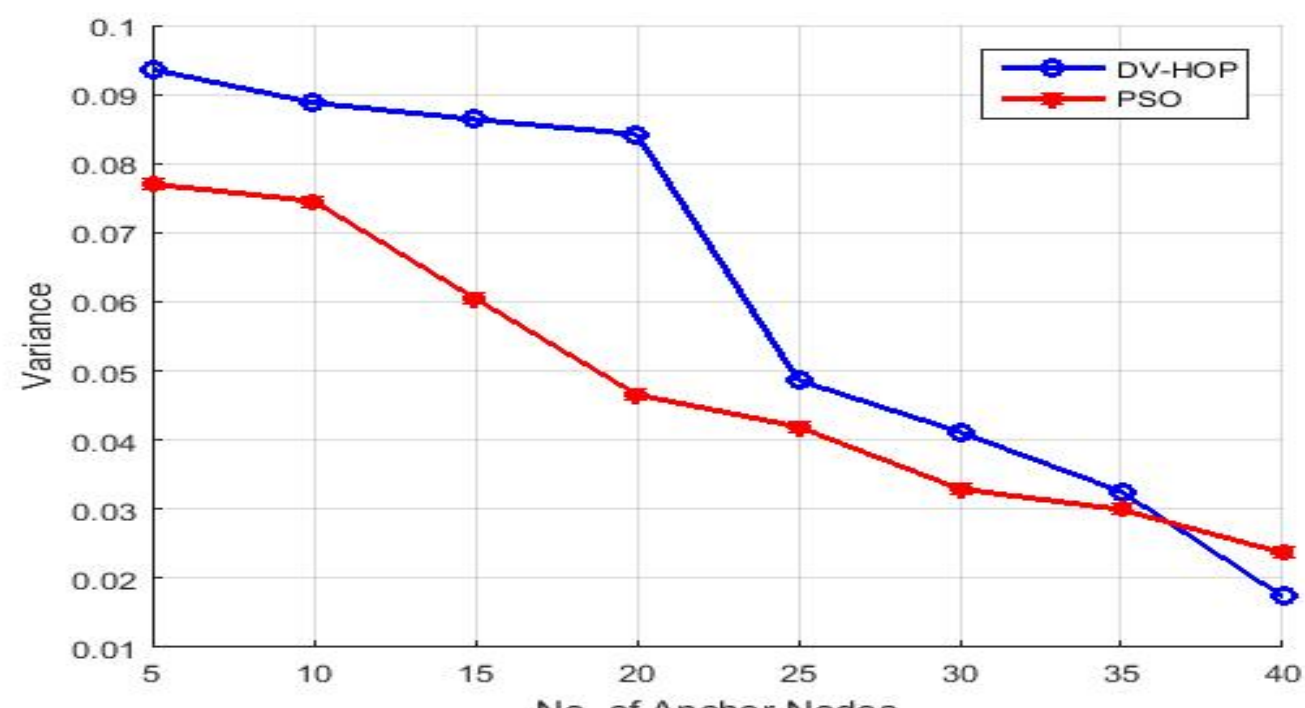

Fig. 8. Localization error variance with changing no. of anchor nodes

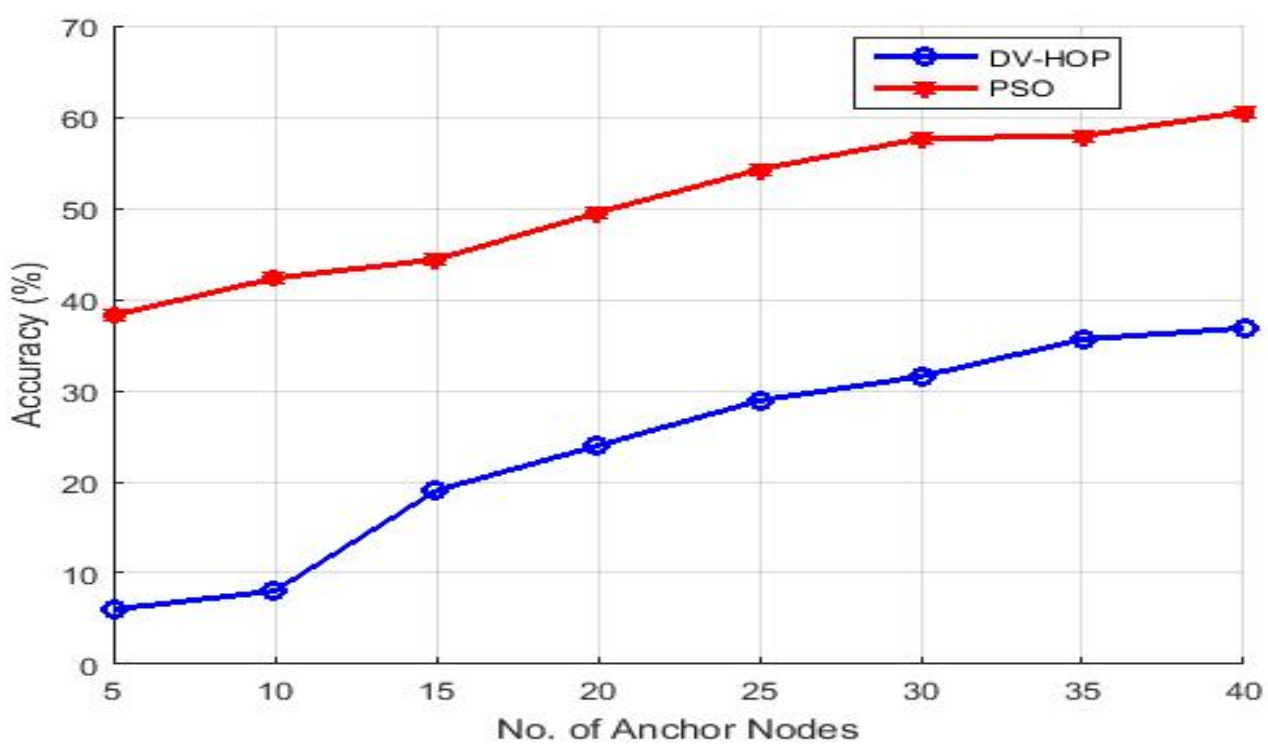

Fig. 9. Localization accuracy with changing no. of anchor nodes

3) Impact of radio range of sensor nodes on localization results

An effect of node's radio range on localization error, error variance and accuracy is given away in Fig. 10, Fig. 11, and Fig. 12 respectively 


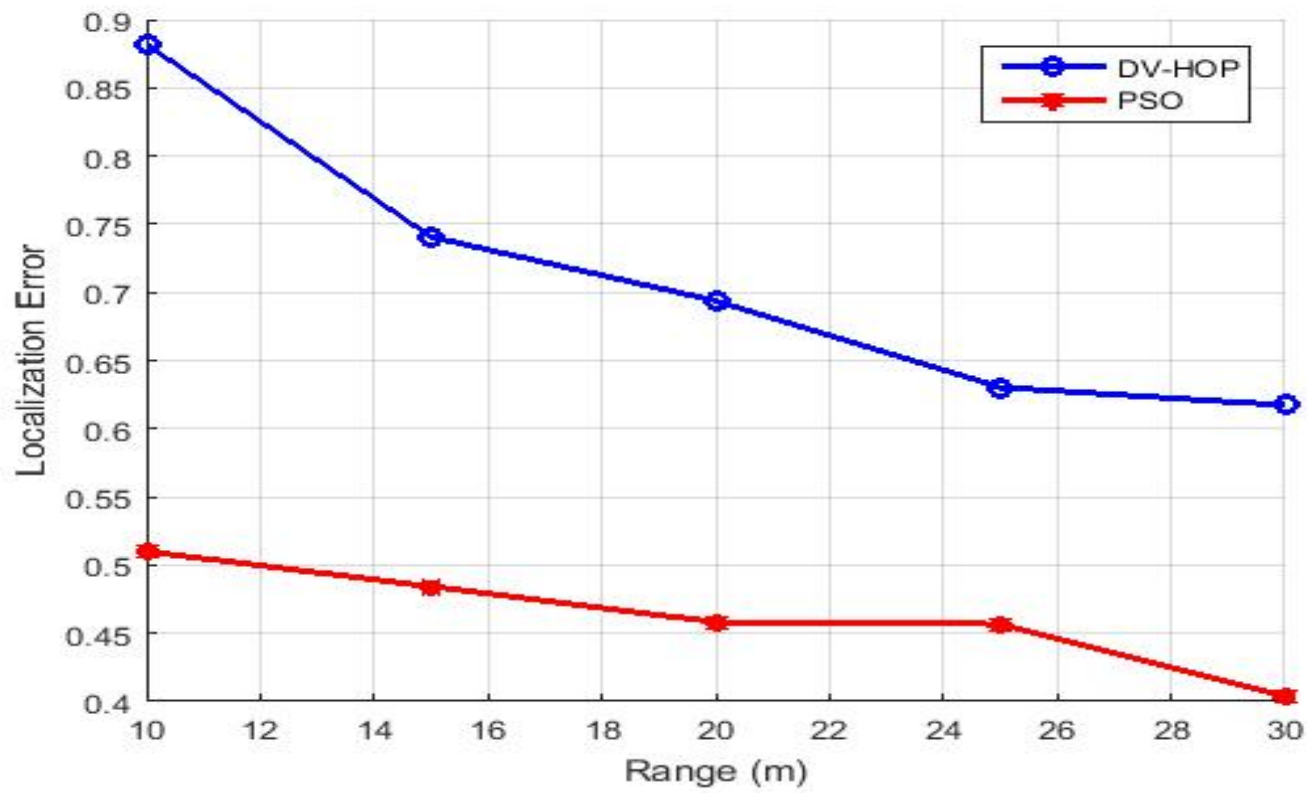

Fig. 10. Localization error with changing communication range

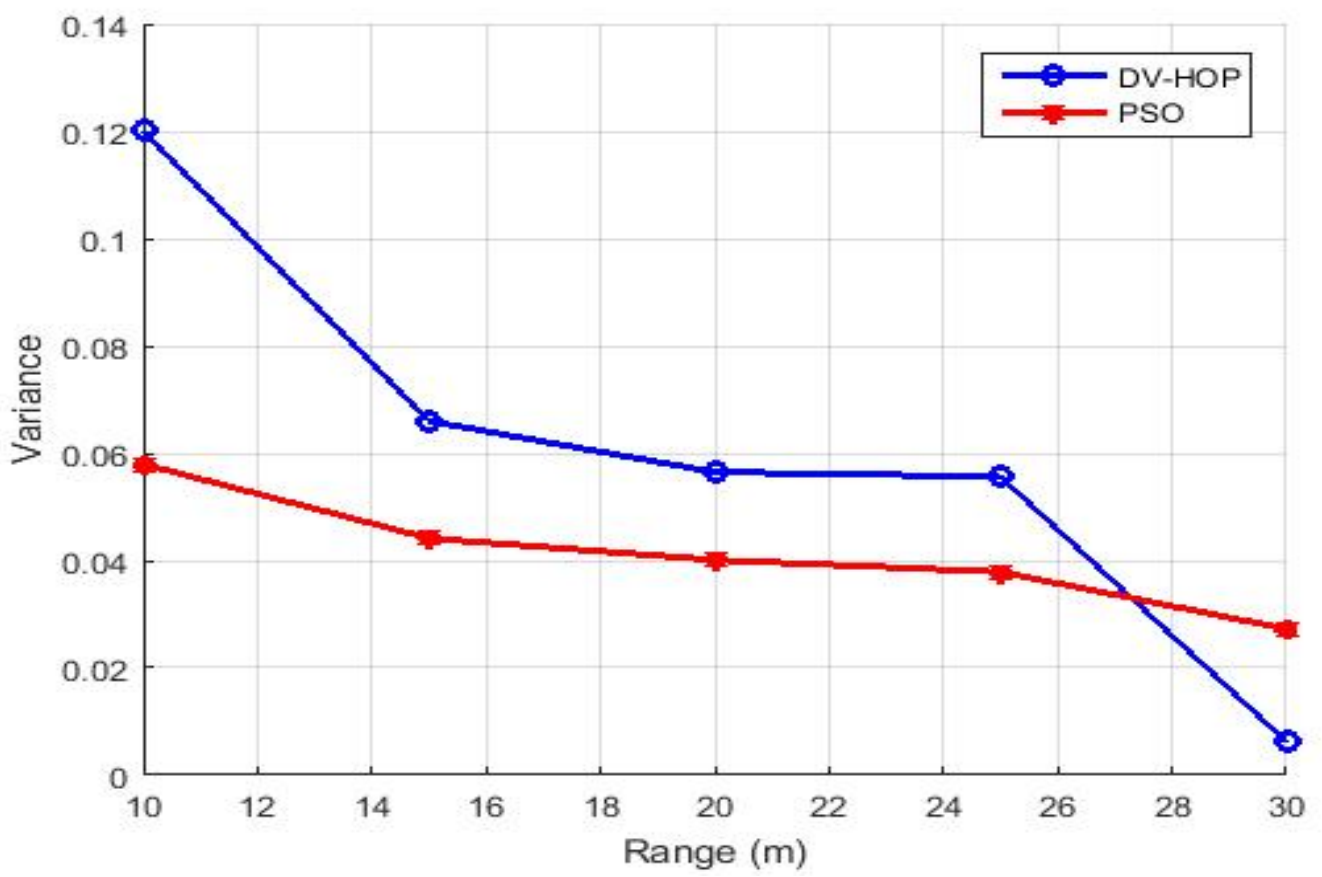

Fig. 11. Localization error variance with changing communication range 


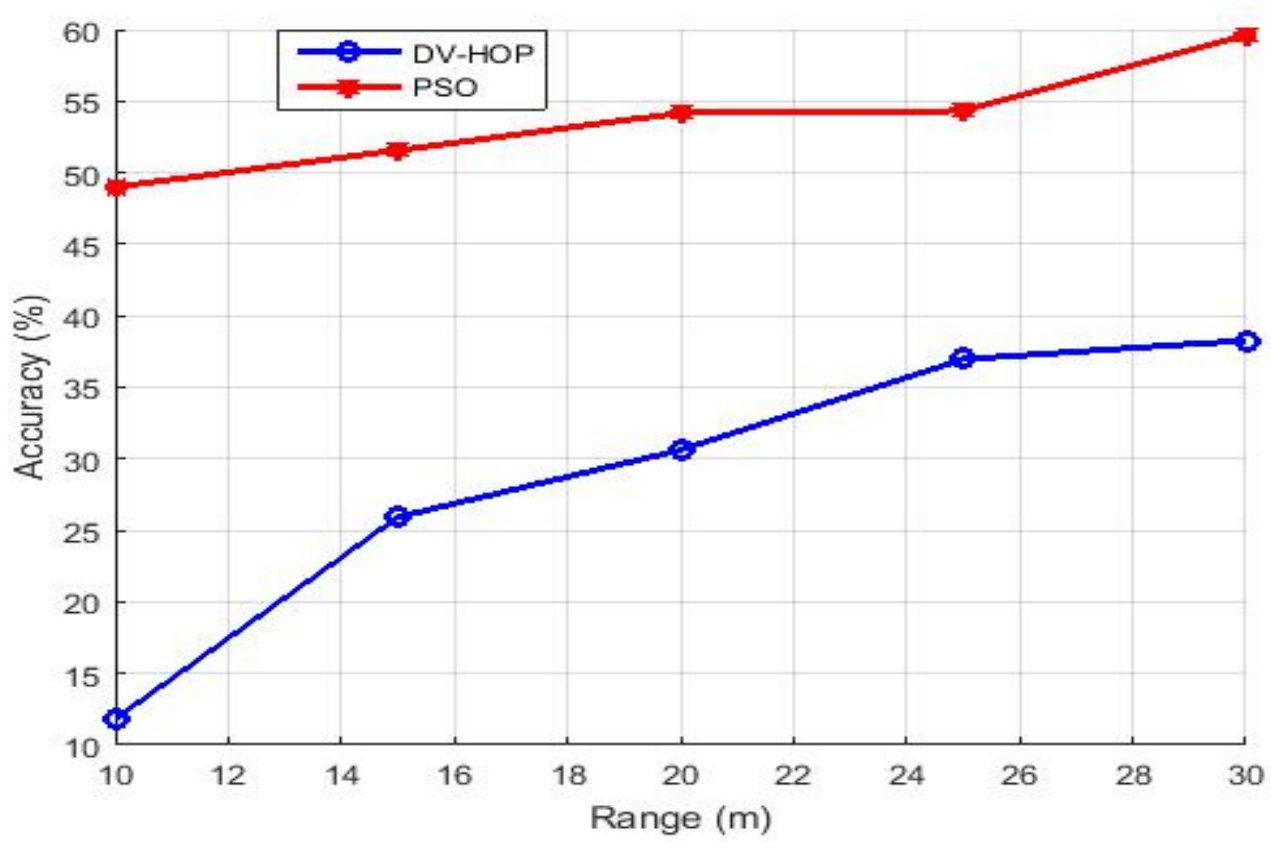

Fig. 12. Localization accuracy with changing communication range

\section{CONCLUSION}

We presented a new algorithm i.e. modified DV-Hop algorithm, which improves the traditional DV-Hop algorithm considerably. The analysis of simulation result section states that our modified algorithm betters the localization error, error variance, accuracy as compared to basic hop based localization algorithm. As revealed in the simulation section of this paper, it can be stated that proposed method is efficient and have excellent application forefront. We also explored the effect of no of sensor nodes, no of anchor nodes, and radio range on the localization error as well as on accuracy. In proposed algorithm, we correct the position estimates with help of PSO. It is clear that modified algorithm boost the precision and stability of localization method. But due to the use of PSO, little increase in computation time.

\section{ACKNOWLEDGMENT}

This work is supported by human resource development (MHRD) ministry under research grant scheme for doctoral work.

\section{REFERENCES}

[1] I. F. Akyildiz, W. Su, Y. Sankarasubramaniam, E. Cyirci, "Wireless sensor networks: a survey," Computer Networks, vol. 38 no.4, pp. 393-422, 2002.

[2] K. Romer, M. Friedemann, "The design space of wireless sensor networks," IEEE Wireless Communications, vol. 11, no.6, pp.54-61, 2004.

[3] K. Sohraby, D. Minoli, T. Znati, "Wireless Sensor Networks: Technology. Protocols, and Applications," NY, USA: Wiley, 2007.

[4] J. Yick, M. Biswanath, D. Ghosal, "Wireless sensor network survey," Computer Networks, vol. 52, no.12, pp. 2292-2330, 2008.

[5] G. Mao, B. Fidan, B. D. Anderson, "Wireless sensor networks localization techniques," Computer Networks, vol. 51, no. 10, pp. 2529-2553, 2007.

[6] W. Jing, R. K. Gosh, S.K., Das, “A survey on sensor localization,” Journal of Control Theory and Applications, vol. 8, no. 1, pp. 2-11, 2010.

[7] A. Samira, "A Review of Localization Techniques for wireless sensor networks," Journal of .Basic and Applied Science Research, vol. 2, no. 8, pp. 795-7801, 2012.

[8] I. Stojmenovic, J. Bachrach, C. Taylor, "Localization in sensor network," in Handbook of Sensor Networks: Algorithms and Architectures, pp.277--310, 2005

[9] L. Yunhao, Y. Zeng, W. Xiaoping, J. Lirong, "A Location, localization and localizability," Journal of Computer Science and Technology, vol. 25, no. 2, pp. 274-297, 2010.

[10] B. H. Wellenhof, H. Lichtenegger, and J. Collins, "Global positioning system: theory and practice", $4^{\text {th }}$ ed, Berlin Germany: Springer, 1997.

[11] J. Bachrach, R. Nagpal, M. Salib, H. Shrobe, "Experimental results and theoritical analysis of a self-organizing a global coordinate system from Ad Hoc sensor networks," Telecommunications System Journal, vol. 26, no.2-4, pp. 213-233, 2004.

[12] G. Han_, H. Xu, T. Q. Duong, J. Jiang, and T. Hara, "Localization algorithms of wireless sensor networks: a survey," Telecommunication Systems, vol. 52, no. 4, pp.2419-2436, April 2013

[13] A. Pal, "Localization algorithms in wireless sensor networks: current approaches and future challenges," Network Protocols and Algorithms, vol. 2, pp.45-73, 2010 
[14] E. Elnahrawy, X. L,i and R. P. Martin, "The limits of localization using signal strength: a comparative study," in Proceedings of IEEE International Conference on Sensing, Communication and Networking (SECON), Santa Clara, California, USA, pp.406-414, 2004

[15] Y. Zhang, W. Wu, Y. Chen, “A Range Based Localization Algorithm for Wireless Sensor Network,” Journal of Communication and Networks, vol.7, no. 5, pp.429-437, 2005.

[16] D. Niculescu, B. Nath, "DV Based Positioning in Ad Hoc Networks," Telecommunications System Journal, vol.22, no.14, pp. 267280, 2003.

[17] T. He, C. Huang, B. Blum, J. Stankovic, T. Abdelzaher, "Range-free localization schemes in large scale sensor networks," in Proceeding of Ninth Annual International Conference on Mobile Computing and Networking (MobiCom'03),pp.81-95, San Diego, CA, USA, 2003.

[18] N. Bulusu, J. Hedimann, D. Estrin, “ GPS-less low-cost outdoor localization for very small device," IEEE Personal Communication, vol.7, no.5, 28-34, 2000.

[19] S. P. Singh, S. C. Sharma, "Range Free Localization Techniques in Wireless Sensor Networks: A Review," Procedia Computer Science vol.57, pp.7-16, 2015.

[20] R. Nagpal, H. Shrobe, J. Bachrach, "Organizing a Global Coordinate System from local information on an Ad Hoc Sensor Networks," in Ninth Annual International Conference on Information Processing in Sensor Networks (ISPN 03),pp.333--348, Palo Alto, CA, USA, 2003.

[21] S. P .Singh, S. C. Sharma, "Critical Analysis of Distributed Localization Algorithms in Wireless Sensor Networks," International Journal of Wireless and Microwave Technologies, vol. 6, no 4, pp.72-83, 2016

[22] R. V. Kulkarni, G. K. Venayagamoorthy, "Particle Swarm Optimization in Wireless Sensor Network: A Brief Survey," IEEE Transactions on System, Man, and Cybernatics-Part C: Applications and Reviews, vol. 41, no.2, pp.262-267, 2011.

[23] S. Tomic, I. Mezei, "Improved DV-Hop Localization Algorithm for Wireless Sensor Networks," in Proceedings of IEEE $10^{\text {th }}$ Jubilee International Symposium on Intelligent Systems and informatics (SISY 2012), Serbia, 2012.

[24] H. Chen, K. Sezaki, K., P. Deng, H. C. Hing, "An Improved DV-Hop Localization Algorithm for Wireless Sensor Networks," in Proceedings of 3rd IEEE Conference on Industrial Electronics and Applications, USA, 2008.

[25] X. Chen, B. Zhang, "An Improved DV-Hop Node Localization Algorithm in Wireless Sensor Networks," International Journal of Distributed Sensor Networks, 2012.

[26] X. Tao et al., "An Improved DV-Hop Node Localization Algorithm Based on Beacon Nodes at Borderland of Wireless Sensor Networks," Lecture Notes in electrical Engineering (LNEE), vol. 143, pp.147-154, 2012.

[27] Y. Hu, X. Li, “An Improvement of DV-Hop Localization Algorithm for Wireless Sensor Networks," Telecommunications System, vol. 53, pp.13-18, 2013.

[28] F. Zhang, "Positioning Research for Wireless Sensor Networks Based on PSO Algorithm," Elekronika Ir Elekronika, vol.19, pp.7-10, 2013.

[29] B. Peong, L. Li, "An improved localization algorithm based on genetic algorithm in wireless sensor networks," Cognitive Neurodynamics, vol.9, no.2, pp.249-256, 2015.

[30] D. Li, B. W. Xian, "An improved PSO algorithm for distributed localization in wireless sensor networks," International Journal of Distributed Sensor Networks, Volume 2015, Article ID 970272.

[31] http://www.mdpi.com/1424-8220/11/5/4721/fig3.

\section{AUTHOR PROFILE}

Santar Pal Singh is student of Ph.D. in Computer Engineering discipline, DPT, Indian Institute of Technology Roorkee (India) since July 2013. He received the B.Tech. in Computer Sc. \& Engineering from Kamla Nehru Institute of Technology, Sultanpur (U.P.) in 2001 and the M.Tech. in Computer Sc. \& Engineering from Samrat Ashok Technological Institute, Vidisha (M.P.) in 2006. His present research interest includes Ad-hoc and wireless sensor networks, network security.

Professor S. C. Sharma received the M.Sc. (Electronics), M.Tech. (Electronics \& Communication Engg.) and Ph.D. (Electronics \& Computer Engg.) from IIT Roorkee (erstwhile University of Roorkee).He has published over two hundred research papers in national and international journals/conferences and supervised more than 30 projects/dissertation of PG students. He has supervised $14 \mathrm{PhDs}$ in the area of Computer Networking, Wireless Network, Computer Communication and continuing supervising Ph.D. students in the same area.

He has successfully completed several major research projects independently funded by various Govt. Agencies like AICTE, CSIR, MHRD, DST, and DRDO Profile about the first author of the manuscript should be updated here. 